\title{
SOLUTION OF ACOUSTIC TOMOGRAPHIC PROBLEMS ON THE BASIS OF NUMERICAL SIMULATION OF PARTICLE DYNAMICS
}

\author{
Angela Kuzovova' ${ }^{I}$, Ivan Kuzmenko \\ Tomsk State University, 634050, Tomsk, Russia
}

\begin{abstract}
A method is proposed for imaging of scattering heterogeneities in continuous media on the basis of numerical modeling of forward and backward wave propagation. It is shown that the combination of a solution for backward propagation of waves and direct wave propagation allows us to visualize scattering heterogeneities. The results of numerical simulation are presented.
\end{abstract}

\section{Introduction}

Acoustic tomography is widely used in flaw detection. To obtain tomographic images, it is necessary to solve the inverse problem - determine its shape and characteristics of object by means of the measured signal scattered by heterogeneity. Inverse problems do not always have a unambiguous solution, and are extremely sensitive to measurement noise and inconsistencies in the mathematical model and sounding conditions of the medium. Most of the existing methods of ultrasonic tomography use the locational principle of sounding $[1,2]$, in which objects are visualized by back scattered waves. There are also transmission schemes for ultrasonic tomography to determine the flat shadow images of the objects under study. Often, the approximation of geometrical optics is used to the solve inverse tomography problems $[3,4]$. The most universal method of processing ultrasound sounding data is the method of spatially-matched filtering. By measuring the field at a variety of different points in space and it is possible to synthesize the aperture and obtain images with a resolution comparable to the wavelength. This method works in the frequency domain and for ultra-wideband signals it is demanding of large computing resources. However, there are particular cases of sounding schemes where rapid processing of signals and obtaining of three-dimensional images is possible, for example, the Stolt method. To process signals in short-pulse sounding, a migration method in the time domain is used, or the method of diffraction hyperbolas summation. This method is quite universal, and can take into account any combination of positions of the emitters and receivers. Such methods are fast-acting, but do not allow to take into account for the influence of background heterogeneities and nonlinear distortions.

In this paper, we propose a method for solving the inverse problem of acoustic tomography based on numerical simulation of the propagation of acoustic waves in solids.

\footnotetext{
${ }^{1}$ Corresponding author: Ang_kuz93@mail
} 


\section{Method of acoustic tomography}

Solid is represented as an array of particles in a tetrahedral equidistant grid. The dynamics of particles is described by Newton's equations of motion. To reconstruct the image of scattering heterogeneities, the time reversal method is used in the numerical model under consideration. In this case, it is assumed that background media heterogeneities are known, but it is necessary to detect unknown defects, for example, in the form of a gap in the crystal lattice.

Let us consider a numerical model of the propagation of acoustic waves in a solid in which the scattering object (heterogeneity) has the form shown in Figure 1, and the background medium is the equivalent of brass (sound velocity $4430 \mathrm{~m} / \mathrm{c}$, density $8485 \mathrm{~kg} / \mathrm{m}^{3}$ ). Heterogeneity it is a gap of the crystal lattice, through which acoustic waves do not pass. We consider the source of the acoustic signal in the form of a plane wave radiated in the forward direction of the $y$ axis. The shape of the original signal is a monopolar pulse in the form of a Gauss curve with a duration of $2.4 \mu$ s (at half-amplitude level). A plane wave, propagating downward in a medium, collides with a scattering heterogeneity, diffracts and reflects. Part of the scattered field is registered by receiving sensors, in which are measured the particle velocity and their deviation in the crystal lattice depending on time. Receiving sensors are located on the plane $y=0$ with a step equal to the step of the particles placement.

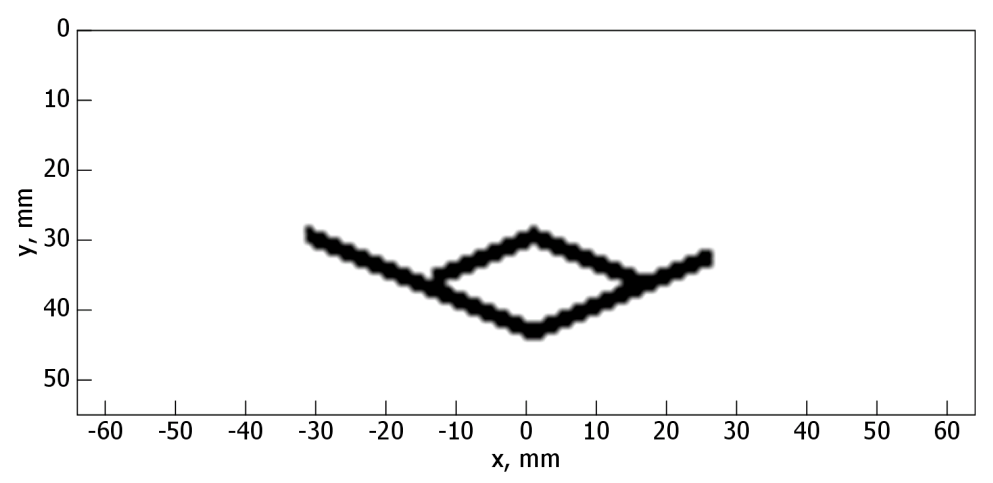

Fig.1. Image of test heterogeneity.

In Figure 2 the image of the field of the scattered wave on the heterogeneity under consideration is presented. One can observe the reflection of the acoustic pulse of a plane wave from the heterogeneity. The incident wave does not pass through the upper part of the heterogeneity, but is completely reflected, hence, the lower part of the heterogeneity will not scatter the field and will not be detected. The all scattered field toward to the side the plane $y=0$ is recorded, namely the velocity vectors and the coordinates of the upper particles.

The solution of the inverse problem is based on the method of backward wave propagation. In work [5] this approach was used to reconstruct images of acoustic wave sources. The method of backward propagation of waves is based on the same process of numerical modeling as the direct problem, but here the time step is taken to be negative. At the same time, the receiving points become emitters, and they radiate precisely that one the signal that was accepted at the appropriate moment of time. 


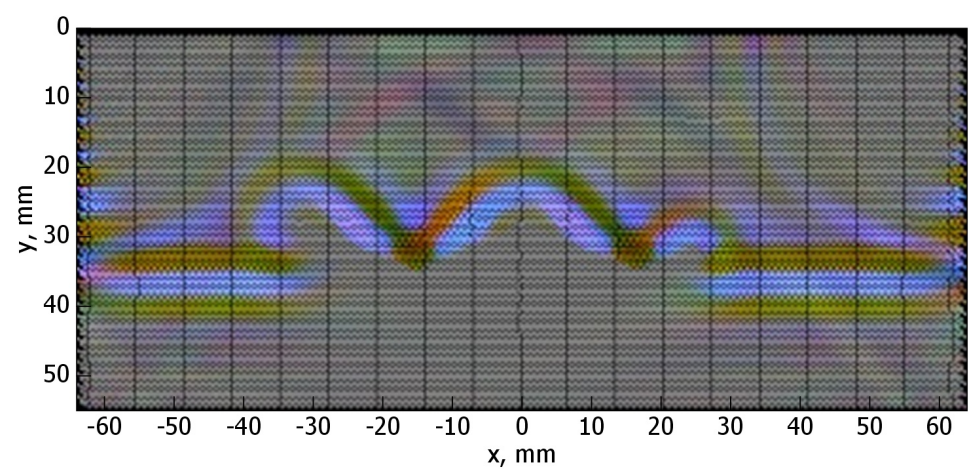

Fig. 2. The solution of a direct problem at a certain moment in time.

The denser the receivers will fill the whole space, the more accurate it will be possible to restore the complete initial state of the system at past moment times. So it will be possible to restore the cause of the appearance of the scattered field. In our case, the receivers are located at the top, so the backward propagation wave will pass from the top to bottom. At the point where the point scatterer is located, the backward propagation wave converges in a point (with a localization region of the order of the pulse length). If continue the reverse course of time already focused field in the scattering point will begin to diverge. It is not known in advance at what point in time it is necessary to stop reverse propagation, so we can not exactly guarantee the restoration of the scatterer. To stop the reverse propagation makes sense exactly at the moment when to the considered point in space reached by a forward sounding wave.

It is proposed to reconstruct the image of scattering heterogeneities by combining the numerical simulation solutions with a positive time course and with a negative time course (Figure 3). Initially we assume that the medium is homogeneous and does not contain scatterers, and we model in it the propagation of a plane wave from a source. Next, we simulate the backward propagation of waves from receivers, reversing the time. We scalar multiply the velocities of particles from two models at coinciding instants of time. In the region where the scatterers are located, the forward and backward waves overlap, when they are superimposed (by scalar multiplication of the particle velocities), it is possible to visualize the scattering object. Since the wave does not penetrate into the heterogeneity, which is a gap of the crystal lattice, we cannot visualize hidden parts.

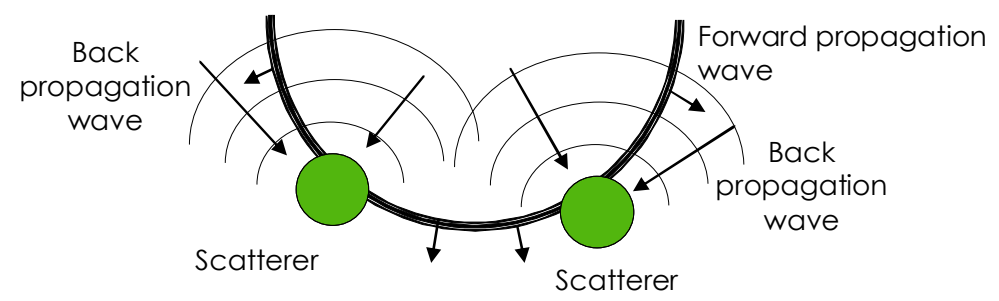

Fig.3. Explanation of the method for solving the inverse problem.

On (Figure 4) is presented the result of the plane wave simulation at back propagation of the field. Red indicates the velocity of the particle along the $X$ axis, green along the $Y$ axis, blue along the $\mathrm{Z}$ axis. The initial perturbation was directed along the $\mathrm{Z}$ axis. It is seen that in the region where the test heterogeneity is located, the field scattered from the object is focused. 


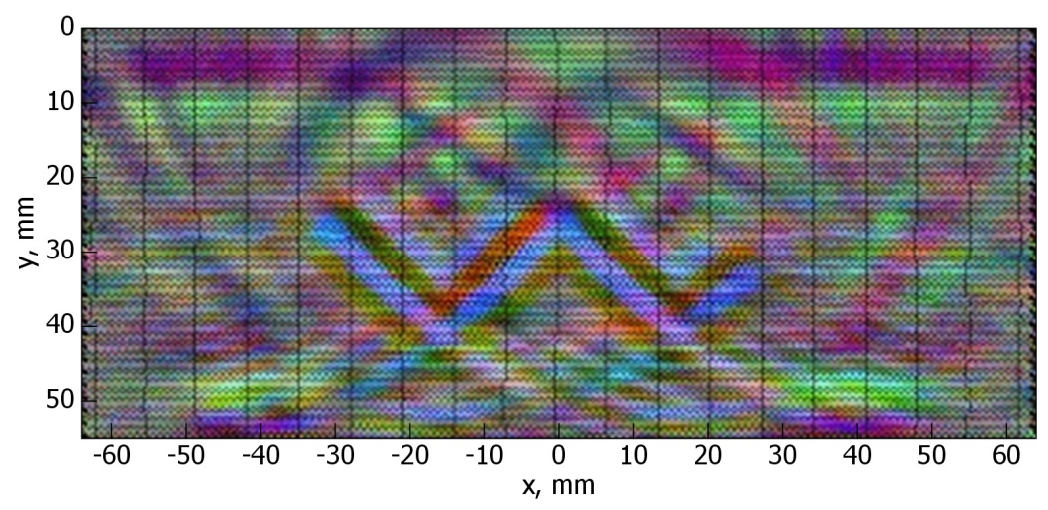

Fig.4. Backward propagation of the field.

Figure 5 shows the reconstructed image of the test heterogeneity. According to the restored result we can determine the shape of the object. The proposed method of image restoration allows to take into account the effect of diffraction. It should be noted that the concave regions and internal voids of objects will not be visualized, since the wave field does not penetrate into these regions.

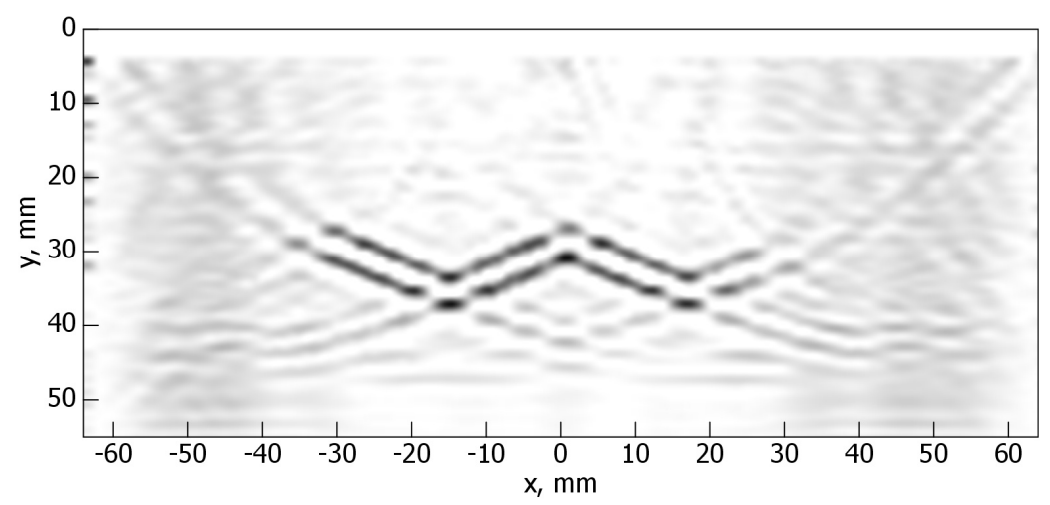

Fig.5. Refurbished image of heterogeneities.

To test the possibility of visualizing objects of more complex shapes, the scatterer was modeled in the form of a circle (Figure 6).

As a result of solving the inverse problem by the proposed method, we have restored the image of the scatterer, shown in Figure 7. It can be seen that only the upper part of the circle is visualized. This is due to the fact that most part of the waves scattered on the object do not return to the side of the receivers. Thus, objects of not any shape can be visualized, it is necessary the presence of waves scattered to the side of the receivers. 


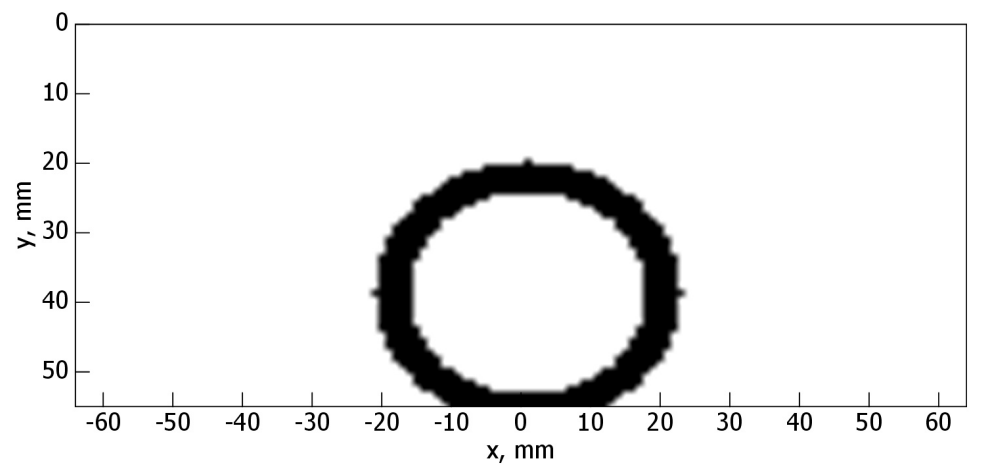

Fig.6. Image of modeled heterogeneity.

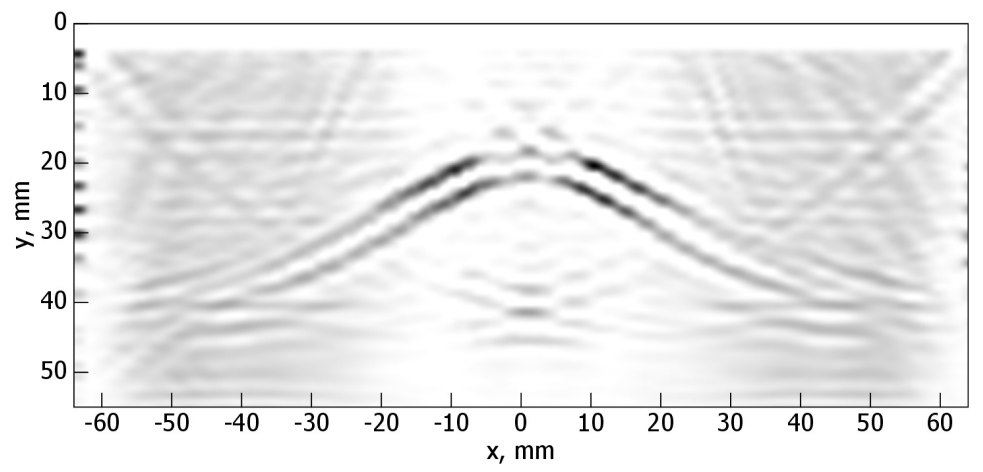

Fig. 7. Refurbished image of heterogeneity.

As a result of the work, the new acoustic topography method is proposed. The method is based on forward and backward time - domain numerical simulation of particles dynamics. It is possible to visualize scattering heterogeneities in a solid media.

\section{Acknowledgments}

The work was supported by the Ministry of Education and Science of Russia within the framework of the project "Creation of a high-tech complex of ultrasound surgery" (Unique identifier of the project RFMEF157517X0163).

\section{References}

[1] A.V. Osetrov, Acoust. Journal 37, 528 ( 1991)

[2] A.V Osetrov, S.N. Samolenkov, Acoust. Journal 42, 679 (1996)

[3] K.J. Opielinski, T. Gudra, Ultrasonics, 295 (2006)

[4] I. Peterlik, R. Jirik, N. Ruiter, J. Jan, Radioengineering, 125 (2008)

[5] D.Ya. Sukhanov, M.A. Kalashnikova, Acoustical Physics 60, 304 (2014) 